\title{
Some Infinite Product Identities
}

\section{By Richard Blecksmith, John Brillhart, and Irving Gerst}

\begin{abstract}
In this paper we derive the power series expansions of four infinite products of the form

$$
\prod_{n \in S_{1}}\left(1-x^{n}\right) \prod_{n \in S_{2}}\left(1+x^{n}\right)
$$

where the index sets $S_{1}$ and $S_{2}$ are specified with respect to a modulus (Theorems 1 , 3 , and 4). We also establish a useful formula for expanding the product of two Jacobi triple products (Theorem 2). Finally, we give nonexistence results for identities of two forms.
\end{abstract}

1. Introduction. During a computer investigation of products of the form

$$
\prod_{\substack{n=1 \\ n \equiv r_{1}, \ldots, r_{t} \\(\bmod m)}}^{\infty}\left(1-x^{n}\right)(\bmod 2)
$$

we found several products with interesting modulo 2 power series expansions. For example, the expansion

$$
\prod_{\substack{n=1 \\ n \not \equiv(\bmod 10)}}^{\infty}\left(1-x^{n}\right) \equiv \sum_{n=0}^{\infty}\left(x^{n(n+1)}+x^{5 n(n+1)+1}\right)(\bmod 2)
$$

is distinctive in that the exponents on the right are quadratic polynomials with different leading coefficients. While attempting to prove these congruences, one of us (IG) discovered with considerable surprise that some of the congruences, such as the one above, are actually equations when the plus and minus signs are chosen properly on the two sides. Four such equations with only minus signs in the factors on the left were proved in [3, Theorems 1 and 2] using standard, single-variable identities derived from the familiar triple and quintuple products. (See (9) and (20) below.)

In the present paper we establish four more equations, but this time with both plus and minus signs in their products (see [4]). For example, the identity

$$
\prod_{\substack{n=1 \\ n=0 . \pm 3 \\(\bmod 10)}}^{\infty}\left(1-x^{n}\right) \prod_{\substack{n \equiv \pm 1, \pm 2, \pm 4 \\(\bmod 10)}}^{\infty}\left(1+x^{n}\right)=\sum_{n=0}^{\infty}\left(x^{n(n+1)}+x^{5 n(n+1)+1}\right)
$$

is the equation standing behind the congruence above. (See Theorem 4.) We note that this equation, as well as (26), (32), and (33), have a partition theory

Received September 25, 1987.

1980 Mathematics Subject Classification (1985 Revision). Primary 05A19.

Key words and phrases. Jacobi triple product, quintuple product, infinite product expansions. 
interpretation as in [8, Chapter 19]. The proofs we give here employ several classical results $((8),(9),(18),(20))$, as well as forms of the quintuple product identity in which both plus and minus signs occur $((21),(23))$. We also develop an expansion formula for the product of two Jacobi triple products (Theorem 2). This interesting result allows us to prove the identities of this paper at quite an elementary level.

2. Preliminaries. We begin with the Jacobi triple product $[8$, p. 283]

$$
\prod_{n=1}^{\infty}\left(1-x^{2 n}\right)\left(1+x^{2 n-1} z\right)\left(1+x^{2 n-1} z^{-1}\right)=\sum_{-\infty}^{\infty} x^{n^{2}} z^{n}
$$

Replacing $(x, z)$ by $\left(x^{k},(-1)^{\varepsilon} x^{l} z^{p}\right), \varepsilon=0$ or 1 , gives

$$
\begin{aligned}
T_{\varepsilon}(k, l ; p) & \stackrel{\text { def }}{=} \prod_{n=1}^{\infty}\left(1-x^{2 k n}\right)\left(1+(-1)^{\varepsilon} x^{2 k n-k+l} z^{p}\right)\left(1+(-1)^{\varepsilon} x^{2 k n-k-l} z^{-p}\right) \\
& =\sum_{-\infty}^{\infty}(-1)^{\varepsilon n} x^{k n^{2}+l n} z^{p n}, \quad k \neq 0 .
\end{aligned}
$$

Replacing $(x, z)$ by $\left(i x^{k},(-1)^{\omega} i x^{l} z^{p}\right), \omega=2$ or 3 , gives

$$
\begin{aligned}
& T_{\omega}(k, l ; p) \stackrel{\text { def }}{=} \prod_{n=1}^{\infty}\left(1-(-1)^{n} x^{2 k n}\right)\left(1+(-1)^{n+\omega} x^{2 k n-k+l} z^{p}\right) \\
& \cdot\left(1+(-1)^{n-1+\omega} x^{2 k n-k-l} z^{-p}\right) \\
&=\sum_{-\infty}^{\infty}(-1)^{\frac{n(n+1)}{2}+\omega n} x^{k n^{2}+l n} z^{p n} .
\end{aligned}
$$

Except for Section 2, we will be dealing with single-variable $T$-functions with $p=0$ :

$$
\begin{aligned}
T_{\varepsilon}(k, l) & \stackrel{\text { def }}{=} \prod_{n=1}^{\infty}\left(1-x^{2 k n}\right)\left(1+(-1)^{\varepsilon} x^{2 k n-k+l}\right)\left(1+(-1)^{\varepsilon} x^{2 k n-k-l}\right) \\
& =\sum_{-\infty}^{\infty}(-1)^{\varepsilon n} x^{k n^{2}+l n}, \quad \varepsilon=0 \text { or } 1,
\end{aligned}
$$

and

$$
\begin{aligned}
T_{\omega}(k, l) & \stackrel{\text { def }}{=} \sum_{n=1}^{\infty}\left(1-(-1)^{n} x^{2 k n}\right)\left(1+(-1)^{n+\omega} x^{2 k n-k+l}\right) \\
\cdot\left(1+(-1)^{n-1+\omega} x^{2 k n-k-i}\right) & \\
= & \sum_{-\infty}^{\infty}(-1)^{\frac{n(n+1)}{2}+\omega n} x^{k n^{2}+l n}, \quad \omega=2 \text { or } 3 .
\end{aligned}
$$

(We only use $T_{\omega}(k, l)$ in Section 6.) Our use of (4) in the sequel will require that the exponents in the products there (and therefore the series) be integers. This implies that $2 k \in \mathbf{Z}-\{0\}, 2 l, k-l \in \mathbf{Z}$. In addition, if the exponents are to be positive, then it is necessary (taking $n= \pm 1$ ) that $2 k>0, k+l>0$, and $k-l>0$. These conditions imply that $|l|<k$, while this latter condition is also clearly sufficient for positivity. (These restrictions on the parameters $k, l$ will be assumed as required without necessarily restating them each time.) Note in this 
case that the exponents in the three factors will run respectively through all the positive integers in the residue classes $0,-k+l, k-l$ modulo $2 k$.

In this paper we are primarily concerned with power series expansions of infinite products of the form $\prod_{n \in S}\left(1 \pm x^{n}\right)$, where the index set $S$ consists of the members of certain residue classes with respect to a modulus $m$. For this purpose, we have come to use the following simplified notation:

$$
\left(r_{1}, \ldots, r_{t}\right)_{m}=\prod_{\substack{n=1 \\ n \equiv r_{1}, \ldots, r_{t}(\bmod m)}}^{\infty}\left(1-x^{n}\right)
$$

and

$$
\left[r_{1}, \ldots, r_{t}\right]_{m}=\prod_{\substack{n=1 \\ n \equiv r_{1}, \ldots, r_{t}(\bmod m)}}^{\infty}\left(1+x^{n}\right) .
$$

where $m \in \mathbf{Z}^{+}$and the $r_{i}$ are residues modulo $m$. (When an $r_{i}$ is repeated in the symbol $s$ times, the corresponding factor is multiplied into the product $s$ times.) Accordingly, in this notation (6) and (7) yield for $|l|<k$ the formulas

$$
\begin{gathered}
T_{0}(k, l)=(0)_{2 k}[ \pm(k-l)]_{2 k}=\sum_{-\infty}^{\infty} x^{k n^{2}+l n} \\
T_{1}(k, l)=(0, \pm(k-l))_{2 k}=\sum_{-\infty}^{\infty}(-1)^{n} x^{k n^{2}+l n} \\
T_{2}(k, l)=(0, \pm(k+l))_{4 k}[ \pm(k-l), 2 k]_{4 k}=\sum_{-\infty}^{\infty}(-1)^{n(n+1) / 2} x^{k n^{2}+l n}
\end{gathered}
$$

and

$$
T_{3}(k, l)=(0, \pm(k-l))_{4 k}[ \pm(k+l), 2 k]_{4 k}=\sum_{-\infty}^{\infty}(-1)^{n(n+3) / 2} x^{k n^{2}+l n} .
$$

Two classical formulas which we shall use (with $x^{k}$ replacing $x$ ), due to Euler and Gauss, respectively [8, p. 282], may be written in our notation as

$$
T_{1}\left(\frac{3 k}{2}, \frac{k}{2}\right)=(0)_{k}=\sum_{-\infty}^{\infty}(-1)^{n} x^{n(3 n+1) k / 2}
$$

and

$$
\frac{1}{2} T_{0}\left(\frac{k}{2}, \frac{k}{2}\right)=\frac{(0)_{2 k}}{(k)_{2 k}}=\sum_{n=0}^{\infty} x^{n(n+1) k / 2} .
$$

It also follows from the product in (4) that

$$
T_{\varepsilon}(k,-l)=T_{\varepsilon}(k, l)
$$

and

$$
T_{1}(k, r k)=0, \quad r \text { odd }
$$

since

$$
T_{1}(k, r k)=\sum_{-\infty}^{(-r-1) / 2}(-1)^{n}\left(x^{k}\right)^{n^{2}+r n}+\sum_{(-r+1) / 2}^{\infty}(-1)^{n}\left(x^{k}\right)^{n^{2}+r n}
$$


and replacing $n$ by $-n-r$ in the first sum gives the negative of the second sum. In particular,

$$
T_{1}(k, \pm k)=0
$$

We will also need the identity

$$
T_{0}(k, l)-T_{1}(k, l)=2 x^{k-l} T_{0}(4 k, 4 k-2 l),
$$

which is proved by splitting the index values in the sums into even and odd parts. We derive

$$
[0]_{k}(k)_{2 k}=1,
$$

from the familiar Euler formula $[0]_{1}(1)_{2}=1$ by replacing $x$ by $x^{k}$. Finally, we obtain four useful single-variable expansions from the familiar quintuple product formula [5]

$$
\begin{aligned}
& \prod_{n=1}^{\infty}\left(1-x^{n}\right)\left(1-x^{n} z\right)\left(1-x^{n-1} z^{-1}\right)\left(1-x^{2 n-1} z^{2}\right)\left(1-x^{2 n-1} z^{-2}\right) \\
& \quad=\sum_{-\infty}^{\infty} x^{\left(3 n^{2}+n\right) / 2}\left(z^{3 n}-z^{-3 n-1}\right)
\end{aligned}
$$

by replacing $(x, z)$ by $\left(x^{m}, x^{-k}\right),\left(-x^{m}, x^{-k}\right),\left(x^{m},-x^{-k}\right)$, and $\left(-x^{m},-x^{-k}\right)$, where $0<2 k<m$ :

$$
\begin{aligned}
Q(m, k) & \stackrel{\text { def }}{=}(0, \pm k, \pm(m-2 k), \pm(m-k), m)_{2 m} \\
& =\sum_{-\infty}^{\infty} x^{m\left(3 n^{2}+n\right) / 2}\left(x^{-3 k n}-x^{3 k n+k}\right)
\end{aligned}
$$

$$
\begin{aligned}
Q_{1}(m, k) & \stackrel{\text { def }}{=}(0, \pm k)_{2 m}[ \pm(m-2 k), \pm(m-k), m]_{2 m} \\
& =\sum_{-\infty}^{\infty}(-1)^{\left(3 n^{2}+n\right) / 2} x^{m\left(3 n^{2}+n\right) / 2}\left(x^{-3 k n}-x^{3 k n+k}\right),
\end{aligned}
$$

$$
\begin{aligned}
Q_{2}(m, k) & \stackrel{\text { def }}{=}(0, \pm(m-2 k), m)_{2 m}[ \pm k, \pm(m-k)]_{2 m} \\
& =(0, \pm(m-2 k), m)_{2 m}[ \pm k]_{m} \\
& =\sum_{-\infty}^{\infty}(-1)^{n} x^{m\left(3 n^{2}+n\right) / 2}\left(x^{-3 k n}+x^{3 k n+k}\right)
\end{aligned}
$$

$$
\begin{aligned}
Q_{3}(m, k) & \stackrel{\text { def }}{=}(0, \pm(m-k))_{2 m}[ \pm k, \pm(m-2 k), m]_{2 m} \\
& =\sum_{-\infty}^{\infty}(-1)^{\left(3 n^{2}-n\right) / 2} x^{m\left(3 n^{2}+n\right) / 2}\left(x^{-3 k n}+x^{3 k n+k}\right) .
\end{aligned}
$$

It immediately follows that

$$
Q(m, k)=T_{0}\left(\frac{3 m}{2}, \frac{m}{2}-3 k\right)-x^{k} T_{0}\left(\frac{3 m}{2}, \frac{m}{2}+3 k\right)
$$

and

$$
Q_{2}(m, k)=T_{1}\left(\frac{3 m}{2}, \frac{m}{2}-3 k\right)+x^{k} T_{1}\left(\frac{3 m}{2}, \frac{m}{2}+3 k\right)
$$




\section{The First Identity.}

THEOREM 1. There holds

$$
\prod_{\substack{n=1 \\ n=0, \pm 5, \pm 7 \\(\bmod 24)}}^{\infty}\left(1-x^{n}\right) \prod_{\substack{n \equiv \pm 1, \pm 4,6 \\(\bmod 12)}}^{\infty}\left(1+x^{n}\right)=\sum_{n=0}^{\infty}\left(x^{2 n(n+1)}+x^{6 n(n+1)+1}\right) .
$$

Proof. Equation (26) can be rewritten as

$$
[0]_{12}(0)_{12}( \pm 5)_{12}[ \pm 1, \pm 4,6]_{12}=\frac{(0)_{8}}{(4)_{8}}+x \frac{(0)_{24}}{(12)_{24}}
$$

by (13), or

$$
[0]_{12} Q_{3}(6,1)=(0)_{8}[0]_{4}+x \frac{(0)_{24}}{(12)_{24}}
$$

by $(23)$ and (18). But $[0]_{12}(12)_{24}=1$ by $(18)$, so if we multiply the above equation by $(12)_{24}$ we obtain

$$
Q_{3}(6,1)=(0)_{8}(12)_{24}[0]_{4}+x(0)_{24} \text {. }
$$

Now

$$
\begin{aligned}
(0)_{8}(12)_{24}[0]_{4} & =(0)_{16}(8)_{16}(12)_{24}[0]_{8}[4]_{8}=(0)_{16}(12)_{24}[4,12,20]_{24} \quad(\text { by }(18)) \\
& =(0,16,32,24)_{48}[4,20]_{24}=(0, \pm 16,24)_{48}[ \pm 4, \pm 20]_{48}=Q_{2}(24,4) .
\end{aligned}
$$

We must therefore show that

$$
Q_{3}(6,1)=Q_{2}(24,4)+x(0)_{24}
$$

From (23) we have

$$
\begin{aligned}
Q_{3}(6,1)= & \sum_{-\infty}^{\infty}(-1)^{\left(3 n^{2}-n\right) / 2}\left(x^{(3 n)^{2}}+x^{(3 n+1)^{2}}\right) \\
= & \sum_{-\infty}^{\infty}\left(x^{(12 n)^{2}}+x^{(12 n+1)^{2}}-x^{(12 n+3)^{2}}-x^{(12 n+4)^{2}}-x^{(12 n+6)^{2}}\right. \\
= & \sum_{-\infty}^{\infty}\left(x^{(12 n)^{2}}+x^{(12 n+1)^{2}}+x^{(12 n+2)^{2}}-x^{(12 n+9)^{2}}+x^{(12 n+10)^{2}}\right) \\
= & \sum_{-\infty}^{\infty}(-1)^{n}\left(x^{(12 n+8)^{2}}\right) \\
= & Q_{2}(24,4)+x(0)_{24},
\end{aligned}
$$

by (22) and (12).

Remark. Replacing $x$ by $-x$ in Theorem 1 gives the identity

$$
\prod_{\substack{n=1 \\ n=0, \pm 1, \pm 11 \\(\bmod 24)}}^{\infty}\left(1-x^{n}\right) \prod_{\substack{n \equiv \pm 4, \pm 5,6 \\(\bmod 12)}}^{\infty}\left(1+x^{n}\right)=\sum_{n=0}^{\infty}\left(x^{2 n(n+1)}-x^{6 n(n+1)+1}\right)
$$

4. An Expansion Formula. We now establish a useful expansion formula for the product of two general $T$-functions defined in (2). The proof of this result generalizes a technique of Carlitz and Subbarao [5]. 
THEOREM 2. Let $\varepsilon_{1}, \varepsilon_{2} \in\{0,1\}, k_{1}, l_{1}, p_{1}, k_{2}, l_{2}, p_{2} \in \mathbf{Z}$ and $m \in \mathbf{Z}^{+}$. If $a$ and $b$ are integers satisfying the separability condition*

$$
k_{1} b=k_{2} a(m-a b)
$$

then

$$
\begin{aligned}
& T_{\varepsilon_{1}}\left(k_{1}, l_{1} ; p_{1}\right) T_{\varepsilon_{2}}\left(k_{2}, l_{2} ; p_{2}\right) \\
& \quad=\sum_{r \in R}(-1)^{\varepsilon_{2} r} x^{k_{2} r^{2}+l_{2} r} z^{p_{2} r} T_{\delta_{1}}\left(K_{1}, L_{1}(r) ; P_{1}\right) T_{\delta_{2}}\left(K_{2}, L_{2}(r) ; P_{2}\right),
\end{aligned}
$$

where

$$
\begin{array}{lll}
K_{1}=k_{1}+k_{2} a^{2}, & L_{1}(r)=l_{1}-l_{2} a-2 k_{2} a r, & P_{1}=p_{1}-p_{2} a, \\
K_{2}=k_{2} m(m-a b), & L_{2}(r)=\left(2 k_{2} r+l_{2}\right)(m-a b)+l_{1} b, & P_{2}=p_{1} b+p_{2}(m-a b),
\end{array}
$$

$$
\begin{gathered}
\delta_{1}= \begin{cases}0 & \text { if } \varepsilon_{1}-\varepsilon_{2} a \text { is even } \\
1 & \text { if } \varepsilon_{1}-\varepsilon_{2} a \text { is odd }\end{cases} \\
\delta_{2}= \begin{cases}0 & \text { if } \varepsilon_{1} b+\varepsilon_{2}(m-a b) \text { is even } \\
1 & \text { if } \varepsilon_{1} b+\varepsilon_{2}(m-a b) \text { is odd },\end{cases}
\end{gathered}
$$

and $R$ is a complete residue system $(\bmod m)$.

Proof. Let

$$
\begin{aligned}
S & =T_{\varepsilon_{1}}\left(k_{1}, l_{1} ; p_{1}\right) T_{\varepsilon_{2}}\left(k_{2}, l_{2} ; p_{2}\right) \\
& =\sum_{i}(-1)^{\varepsilon_{1} i} x^{k_{1} i^{2}+l_{1} i} z^{p_{1} i} \sum_{j}(-1)^{\varepsilon_{2} j} x^{k_{2} j^{2}+l_{2} j} z^{p_{2} j} .
\end{aligned}
$$

The change of index $j=n-a i$, which sets up a 1-1 correspondence between $\{(i, j)\}$ and $\{(i, n)\}$, gives

$$
\begin{gathered}
S=\sum_{i}(-1)^{\left(\varepsilon_{1}-\varepsilon_{2} a\right) i} x^{\left(k_{1}+k_{2} a^{2}\right) i^{2}+\left(l_{1}-l_{2} a\right) i} z^{\left(p_{1}-p_{2} a\right) i} \\
\cdot \sum_{n}(-1)^{\varepsilon_{2} n} x^{k_{2} n^{2}+\left(l_{2}-2 k_{2} a i\right) n} z^{p_{2} n}
\end{gathered}
$$

For each $r \in R$, let $S_{r}$ denote the subseries obtained from $S$ as $n$ runs through the set of values congruent to $r$ modulo $m$, so that

$$
S=\sum_{r \in R} S_{r}
$$

To determine $S_{r}$, put $n=s m+r$, where $s$ is a new summation index, obtaining

$$
\begin{aligned}
S_{r}= & (-1)^{\varepsilon_{2} r} x^{k_{2} r^{2}+l_{2} r} z^{p_{2} r} \sum_{i}(-1)^{\left(\varepsilon_{1}-\varepsilon_{2} a\right) i} x^{\left(k_{1}+k_{2} a^{2}\right) i^{2}+\left(l_{1}-l_{2} a-2 k_{2} a r\right) i} z^{\left(p_{1}-p_{2} a\right) i} \\
& \cdot \sum_{s}(-1)^{\varepsilon_{2} m s} x^{k_{2} m^{2} s^{2}+\left(2 k_{2} m r+l_{2} m-2 k_{2} a m i\right) s} z^{p_{2} m s} .
\end{aligned}
$$

* This condition assures that a certain doubly indexed sum in the proof will factor into a product of two singly indexed sums. 
Making a last change of index $i=t+b s$ and setting $d=m-a b$, we obtain

$$
\begin{aligned}
S_{r}= & (-1)^{\varepsilon_{2} r} x^{k_{2} r^{2}+l_{2} r} z^{p_{2} r} \\
& \cdot \sum_{s}(-1)^{\left(\varepsilon_{1} b+\varepsilon_{2} d\right) s} x^{\left(k_{1} b^{2}+k_{2} d^{2}\right) s^{2}+\left(\left(2 k_{2} r+l_{2}\right) d+l_{1} b\right) s} z^{\left(p_{1} b+p_{2} d\right) s} \\
& \cdot \sum_{t}(-1)^{\left(\varepsilon_{1}-\varepsilon_{2} a\right) t} x^{\left(k_{1}+k_{2} a^{2}\right) t^{2}+\left(l_{1}-l_{2} a-2 k_{2} a r\right) t+2\left(k_{1} b-k_{2} a d\right) s t} z^{\left(p_{1}-p_{2} a\right) t} .
\end{aligned}
$$

Using the separability condition (28), the coefficient of $s^{2}$ simplifies and the $s t$ term drops out, giving a sum which separates into the product of two sums. Reversing the order of the sums and using (30) and (31) yields the theorem.

Remarks. 1. It is worth mentioning that (28) can always be satisfied, e.g., $a=k_{1}$, $b=k_{2}, m=k_{1} k_{2}+1$. If a certain value of $m$ is desired, however, then (28) may not be solvable. It can also happen, because of the asymmetry of (28) in $a$ and $b$, that condition (28) may be solvable for some value of $m$ when the factors on the left of (29) are taken in one order but not in the other.

2. The Carlitz and Subbarao proof [5] of the quintuple product formula (19) is a special case of Theorem 2: Replacing $x$ by $x^{2}$ on the left-hand side of (19) and multiplying by $\prod_{n=1}^{\infty}\left(1-x^{4 n}\right)$ gives $T_{1}(2,0 ; 2) T_{1}(1,1 ; 1)$. Applying Theorem 2 with $k_{1}=2, l_{1}=0, p_{1}=2$ and $k_{2}=l_{2}=p_{2}=1$, the separability condition is satisfied with $a=2, b=1$, and $m=3$. Taking $R=\{0,1,-1\}$, we find, after some simplification, that

$$
\begin{aligned}
T_{1}(2,0 ; 2) T_{1}(1,1 ; 1) & =T_{1}(6,2)\left[T_{0}(3,1 ; 3)-z^{-1} T_{0}(3,-1 ; 3)\right] \\
& =(0)_{4} \sum_{-\infty}^{\infty} x^{3 n^{2}+n}\left(z^{3 n}-z^{-3 n-1}\right) .
\end{aligned}
$$

Cancelling $(0)_{4}=\prod_{n=1}^{\infty}\left(1-x^{4 n}\right)$ and replacing $x$ by $x^{1 / 2}$ yields (19).

3. Formula (29) gives an immediate proof of Theorem 1 in [6] and identity (2) in [6], viz., with $m=2, a=b=1$, and $R=\{0,1\}$, we have

$$
\begin{aligned}
T_{0}^{2}(1,0 ; 1) & =\sum_{r \in R} x^{r^{2}} z^{t} T_{0}(2,-2 r ; 0) T_{0}(2,2 r ; 2) \\
& =\sum_{-\infty}^{\infty} x^{2 n^{2}} \sum_{-\infty}^{\infty} x^{2 n^{2}} z^{2 n}+x z \sum_{-\infty}^{\infty} x^{2 n^{2}+2 n} \sum_{-\infty}^{\infty} x^{2 n^{2}+2 n} z^{2 n} .
\end{aligned}
$$

\section{Three More Identities.}

THEOREM 3. We have

$$
\prod_{\substack{n=1 \\ n=0, \pm 1 \\(\bmod 10)}}^{\infty}\left(1-x^{n}\right) \prod_{\substack{n=1 \\ n= \pm 12 \\(\bmod 40)}}^{\infty}\left(1-x^{n}\right) \prod_{\substack{n=1 \\ n \equiv \pm 4 \\(\bmod 20)}}^{\infty}\left(1+x^{n}\right)=1+\sum_{n=1}^{\infty}(-1)^{n}\left(x^{n^{2}}+x^{5 n^{2}}\right)
$$

and

$$
\begin{gathered}
\prod_{\substack{n=1 \\
n=0, \pm 3 \\
(\bmod 10)}}^{\infty}\left(1-x^{n}\right) \prod_{\substack{n=1 \\
n \equiv \pm 4 \\
(\bmod 40)}}^{\infty}\left(1-x^{n}\right) \prod_{\substack{n=1 \\
n \equiv \pm 8 \\
(\bmod 20)}}^{\infty}\left(1+x^{n}\right) \\
=\sum_{n=1}^{\infty}(-1)^{n}\left(x^{5 n^{2}-1}-x^{n^{2}-1}\right) .
\end{gathered}
$$


Proof. First rewrite (32) and (33) as

$$
\sum_{-\infty}^{\infty}(-1)^{n}\left(x^{n^{2}}+x^{5 n^{2}}\right)=2(0, \pm 1)_{10}( \pm 12)_{40}[ \pm 4]_{20}
$$

and

$$
\sum_{-\infty}^{\infty}(-1)^{n}\left(x^{5 n^{2}}-x^{n^{2}}\right)=2 x(0, \pm 3)_{10}( \pm 4)_{40}[ \pm 8]_{20} .
$$

Subtracting and adding these equations gives

$$
\begin{aligned}
& T_{1}(1,0)=(0, \pm 1)_{10}( \pm 12)_{40}[ \pm 4]_{20}-x(0, \pm 3)_{10}( \pm 4)_{40}[ \pm 8]_{20} \\
& T_{1}(5,0)=(0, \pm 1)_{10}( \pm 12)_{40}[ \pm 4]_{20}+x(0, \pm 3)_{10}( \pm 4)_{40}[ \pm 8]_{20}
\end{aligned}
$$

Multiplying these by $(0)_{4}\left(=T_{1}(6,2)\right.$ by $\left.(12)\right)$ and manipulating the factors on the right puts the identities into the form in which we will prove them, namely

$$
\begin{aligned}
& T_{1}(6,2) T_{1}(1,0)=T_{1}(10,2) Q(10,1)-x T_{1}(10,6) Q(10,3), \\
& T_{1}(6,2) T_{1}(5,0)=T_{1}(10,2) Q(10,1)+x T_{1}(10,6) Q(10,3) .
\end{aligned}
$$

To prove (34), we use Theorem 2 with $p_{1}=p_{2}=0, k_{1}=6, l_{1}=2, k_{2}=1, l_{2}=0$, $\varepsilon_{1}=\varepsilon_{2}=1$. In this case the choice of parameters $a=2, b=1, m=5$, satisfies the separability condition (28), from which we find $\delta_{1}=1, \delta_{2}=0$. It is also convenient to take $R=\{0,1,2,-1,-2\}$. Then

$$
\begin{aligned}
T_{1}(6,2) & T_{1}(1,0)=\sum_{r \in R}(-1)^{r} x^{r^{2}} T_{1}(10,2-4 r) T_{0}(15,2+6 r) \\
= & T_{1}(10,2) T_{0}(15,2)-x T_{1}(10,-2) T_{0}(15,8)+x^{4} T_{1}(10,-6) T_{0}(15,14) \\
& -x T_{1}(10,6) T_{0}(15,-4)+x^{4} T_{1}(10,10) T_{0}(15,-10) \\
= & T_{1}(10,2)\left[T_{0}(15,2)-x T_{0}(15,8)\right]-x T_{1}(10,6)\left[T_{0}(15,4)-x^{3} T_{0}(15,14)\right] \\
= & T_{1}(10,2) Q(10,1)-x T_{1}(10,6) Q(10,3),
\end{aligned}
$$

using (14), (16), and (24). This verifies (34).

We next establish the equation

$$
(0)_{20} T_{1}(5,0)=T_{1}(5,4) Q_{2}(20,4)+x T_{1}(5,2) Q_{2}(20,8) \text {. }
$$

(Note that (35) follows directly from (36) by multiplying each term of (36) by $( \pm 4, \pm 8)_{20}$.) To do this, we first replace $x$ by $x^{5}$ in (34). Since the left-hand sides of the resulting equation and (36) are the same, to prove (36) it suffices to show their right-hand sides are equal, namely

$$
\begin{aligned}
& T_{1}(5,4) Q_{2}(20,4)+x T_{1}(5,2) Q_{2}(20,8) \\
& =T_{1}(50,10) Q(50,5)-x^{5} T_{1}(50,30) Q(50,15) .
\end{aligned}
$$

If in the expression $T_{1}(5,4) Q_{2}(20,4)+x T_{1}(5,2) Q_{2}(20,8)$ we expand the two $Q_{2}$ 's by (25), we obtain

$$
\begin{aligned}
& T_{1}(30,2) T_{1}(5,4)+x^{4} T_{1}(30,22) T_{1}(5,4) \\
& \quad+x T_{1}(30,-14) T_{1}(5,2)+x^{9} T_{1}(30,-34) T_{1}(5,2) .
\end{aligned}
$$

(Here the order of the factors in each term is carefully chosen, as are the signs in the second components. (Cf. (14).)) We next expand the four $T_{1} \cdot T_{1}$ pairs by Theorem 2 with $p_{1}=p_{2}=0$, noting that $k_{1}=30, k_{2}=5$, and $\varepsilon_{1}=\varepsilon_{2}=1$ in each 
pair. In addition, in each of these expansions we take $m=5, R=\{0,1,2,-1,-2\}$, and $a=3, b=1$ so that (28) is satisfied and $\delta_{1}=0, \delta_{2}=1$. It is convenient to exhibit (29) with these values inserted, namely

$$
\begin{aligned}
& T_{1}\left(30, l_{1}\right) T_{1}\left(5, l_{2}\right) \\
& \quad=\sum_{r \in R}(-1)^{r} x^{5 r^{2}+l_{2} r} T_{0}\left(75, l_{1}-3 l_{2}-30 r\right) T_{1}\left(50,20 r+l_{1}+2 l_{2}\right) .
\end{aligned}
$$

Now in (39), if we take $\left(l_{1}, l_{2}\right)$ in turn to be $(2,4),(22,4),(-14,2)$, and $(-34,2)$, we get 20 products of the form $x^{n} T_{0} T_{1}$ of which 16 miraculously cancel when they are properly grouped. To see this, we first note that four terms are 0 , using (16). If the sum of the 16 remaining terms is then separated into 5 subsums $S_{i}, 0 \leq i \leq 4$, where a term $x^{n} T_{0} \cdot T_{1}$ is placed in $S_{i}$ if $n \equiv i(\bmod 5)$, we find that $S_{1}=S_{4}=0$, using (14). We also discover that

$$
S_{2}=x^{12} T_{0}(75,50) T_{1}(50,-30)+x^{32} T_{0}(75,-50) T_{1}(50,70)=0,
$$

because

$$
\begin{aligned}
& x^{32} T_{1}(50,-70)=x^{12} \sum_{-\infty}^{\infty}(-1)^{n} x^{50 n^{2}-70 n+20} \\
& \quad=-x^{12} \sum_{-\infty}^{\infty}(-1)^{n} x^{50 n^{2}+30 n}=-x^{12} T_{1}(50,30) \quad(n \rightarrow n+1) .
\end{aligned}
$$

Next,

$$
S_{3}=-x^{8} T_{0}(75,-50) T_{1}(50,10)+x^{33} T_{0}(75,-100) T_{1}(50,10)=0,
$$

because

$$
x^{33} T_{0}(75,-100)=x^{8} \sum_{-\infty}^{\infty} x^{75 n^{2}-100 n+25}=x^{8} \sum_{-\infty}^{\infty} x^{75 n^{2}+50 n}=x^{8} T_{0}(75,50) .
$$

Finally,

$$
\begin{aligned}
S_{0}= & T_{0}(75,-10) T_{1}(50,10)-x^{5} T_{0}(75,40) T_{1}(50,10)+x^{25} T_{0}(75,-80) T_{1}(50,30) \\
& +x^{25} T_{0}(75,20) T_{1}(50,-70) .
\end{aligned}
$$

But

$$
x^{25} T_{0}(75,-80)=x^{20} \sum_{-\infty}^{\infty} x^{75 n^{2}-80 n+5}=x^{20} \sum_{-\infty}^{\infty} x^{75 n^{2}+70 n}=x^{20} T_{0}(75,70),
$$

and by (40)

$$
x^{25} T_{1}(50,-70)=-x^{5} T_{1}(50,30)
$$

so

$$
\begin{aligned}
S_{0}= & T_{1}(50,10)\left[T_{0}(75,10)-x^{5} T_{0}(75,40)\right] \\
& -x^{5} T_{1}(50,30)\left[T_{0}(75,20)-x^{15} T_{0}(75,70)\right] \\
= & T_{1}(50,10) Q(50,5)-x^{5} T_{1}(50,30) Q(50,15),
\end{aligned}
$$

which is the desired right-hand side of (37). 
THEOREM 4. There holds

$$
\prod_{\substack{n=1 \\ n=0 . \pm 3 \\(\bmod 10)}}^{\infty}\left(1-x^{n}\right) \prod_{\substack{n \equiv \pm 1, \pm 2, \pm 4 \\(\bmod 10)}}^{\infty}\left(1+x^{n}\right)=\sum_{n=0}^{\infty}\left(x^{n(n+1)}+x^{5 n(n+1)+1}\right) .
$$

Proof. The result can also be written

$$
2(0, \pm 3)_{10}[ \pm 1, \pm 2, \pm 4]_{10}=\sum_{-\infty}^{\infty}\left(x^{n(n+1)}+x^{5 n(n+1)+1}\right) .
$$

Replacing $x$ by $x^{4}$ and multiplying by $x$ gives

$$
\sum_{-\infty}^{\infty}\left(x^{(2 n+1)^{2}}+x^{5(2 n+1)^{2}}\right)=2 x(0, \pm 12)_{40}[ \pm 4, \pm 8, \pm 16]_{40},
$$

the form in which we prove Theorem 4 . Now (32) can be rewritten as

$$
\begin{aligned}
2(0, \pm 1)_{10}( \pm 12)_{40}[ \pm 4]_{20}=\sum_{-\infty}^{\infty}(-1)^{n}\left(x^{n^{2}}+x^{5 n^{2}}\right) \\
=\sum_{-\infty}^{\infty}\left(x^{(2 n)^{2}}+x^{5(2 n)^{2}}\right)-\sum_{-\infty}^{\infty}\left(x^{(2 n+1)^{2}}+x^{5(2 n+1)^{2}}\right) .
\end{aligned}
$$

Replacing $x$ by $-x$ in (42) gives

$$
\begin{aligned}
2(0)_{10}( \pm 12)_{40}[ \pm 1]_{10}[ \pm 4]_{20}= & \sum_{-\infty}^{\infty}\left(x^{n^{2}}+x^{5 n^{2}}\right) \\
= & \sum_{-\infty}^{\infty}\left(x^{(2 n)^{2}}+x^{5(2 n)^{2}}\right)+\sum_{-\infty}^{\infty}\left(x^{(2 n+1)^{2}}+x^{5(2 n+1)^{2}}\right)
\end{aligned}
$$

Subtracting (42) from (43) and using (8), (9), and (17) gives

$$
\begin{aligned}
\sum_{-\infty}^{\infty}( & \left.x^{(2 n+1)^{2}}+x^{5(2 n+1)^{2}}\right) \\
& =(0)_{10}( \pm 12)_{40}[ \pm 1]_{10}[ \pm 4]_{20}-(0, \pm 1)_{10}( \pm 12)_{40}[ \pm 4]_{20} \\
& =( \pm 12)_{40}[ \pm 4]_{20}\left\{(0)_{10}[ \pm 1]_{10}-(0, \pm 1)_{10}\right\} \\
& =( \pm 12)_{40}[ \pm 4]_{20}\left\{T_{0}(5,4)-T_{1}(5,4)\right\} \\
& =( \pm 12)_{40}[ \pm 4]_{20} 2 x T_{0}(20,12)=2 x(0, \pm 12)_{40}[ \pm 4, \pm 8, \pm 16]_{40} .
\end{aligned}
$$

Remark. Replacing $x$ by $-x$ in Theorem 4 gives the formula

$$
\prod_{\substack{n=1 \\ n=0, \pm 1 \\(\bmod 10)}}^{\infty}\left(1-x^{n}\right) \prod_{\substack{n=1 \\ n \equiv \pm 2, \pm 3, \pm 4 \\(\bmod 10)}}^{\infty}\left(1+x^{n}\right)=\sum_{n=0}^{\infty}\left(x^{n(n+1)}-x^{5 n(n+1)+1}\right) .
$$

6. Two Nonexistence Results. Since Theorems 1(a) and 3(a) in [3], as well as (32) above, have the form

$$
\prod_{n=1}^{\infty}\left(1+\gamma_{n} x^{n}\right)=1+\sum_{n=1}^{\infty}(-1)^{n}\left(x^{n^{2}}+x^{k n^{2}}\right)
$$


for $k=2,3$, or 5 and certain $\gamma_{n}=0, \pm 1$ for each $n \geq 1$, it is natural to ask whether there are any other values of $k \geq 1$ for which (44) holds. We will answer this question for the more general equation

$$
\prod_{n=1}^{\infty}\left(1+\gamma_{n} x^{n}\right)=1+\sum_{n=1}^{\infty}\left(\delta_{n} x^{n^{2}}+\varepsilon_{n} x^{k n^{2}}\right),
$$

where $\gamma_{n}=0, \pm 1$ and $\delta_{n}, \varepsilon_{n}= \pm 1$ for each $n \geq 1$. Using a simple search algorithm, which runs quickly on an IBM PC, we discover that (45) can hold only when $k=2,3,5$, or 9 .

This search algorithm relies on the function DeadendDegree, which attempts to factor the series $1+\sum_{n=1}^{L} \alpha_{n} x^{n}$ (stored as an array $\left(\alpha_{1}, \ldots, \alpha_{L}\right)$ ) into a product of the form on the left-hand side of (45) up to the specified degree $L$. If no factorization is possible, the program returns the degree at which a contradiction is first obtained. Otherwise, the value $L+1$ is returned. For example, the series

$$
f(x)=1-x+x^{4}-x^{7}+x^{9}+x^{16}+x^{25}-x^{28}+x^{36}
$$

whose exponents are of the form $n^{2}$ and $7 n^{2}$, factors uniquely up to degree 29 as

$$
\begin{array}{r}
p(x)=(1-x)\left(1+x^{4}\right)\left(1+x^{5}\right)\left(1+x^{6}\right)\left(1+x^{12}\right)\left(1+x^{13}\right)\left(1+x^{14}\right)\left(1+x^{16}\right) \\
\left(1-x^{18}\right)\left(1+x^{24}\right)\left(1+x^{26}\right)\left(1+x^{27}\right)\left(1-x^{29}\right) .
\end{array}
$$

However, when this product is multiplied out, we find that

$$
p(x)=1-x+x^{4}-x^{7}+x^{9}+x^{16}+x^{25}-x^{28}+2 x^{30}+x^{31}+\cdots .
$$

The discrepancy of 2 between the coefficients of $x^{30}$ in $p(x)$ and $f(x)$ guarantees that the factorization of the original series $f(x)$ into the form on the left-hand side of (45) has reached a dead end at degree 30. DeadendDegree uses an auxiliary array $\beta(x)=1+\sum_{n=1}^{L} \beta_{n} x^{n}$ :

$$
\text { function DeadEndDegree }\left(L, 1+\sum_{n=1}^{L} \alpha_{n} x^{n}\right)
$$

begin

set $\beta(x)=1$

for $n=1$ to $L$ do

begin

$$
\begin{aligned}
& \delta_{n}=\alpha_{n}-\beta_{n} \\
& \text { if }\left|\delta_{n}\right| \geq 2 \text { then return }(n) \\
& \beta(x)=\beta(x)\left(1+\delta_{n} x^{n}\right)
\end{aligned}
$$

end

$$
\text { return }(L+1)
$$

end.

Given $L$ and a sequence $s=\left\{s_{1}, \ldots, s_{t}\right\}, 1 \leq s_{i} \leq L$, the intermediate function LargestDegree returns the value

$$
\max _{\substack{\varepsilon_{i}=1 \text { or }-1 \\ 1 \leq i \leq t}} \text { DeadendDegree }\left(L, 1+\sum_{i=1}^{t} \varepsilon_{i} x^{s_{i}}\right) \text {. }
$$


The search algorithm can now be described:

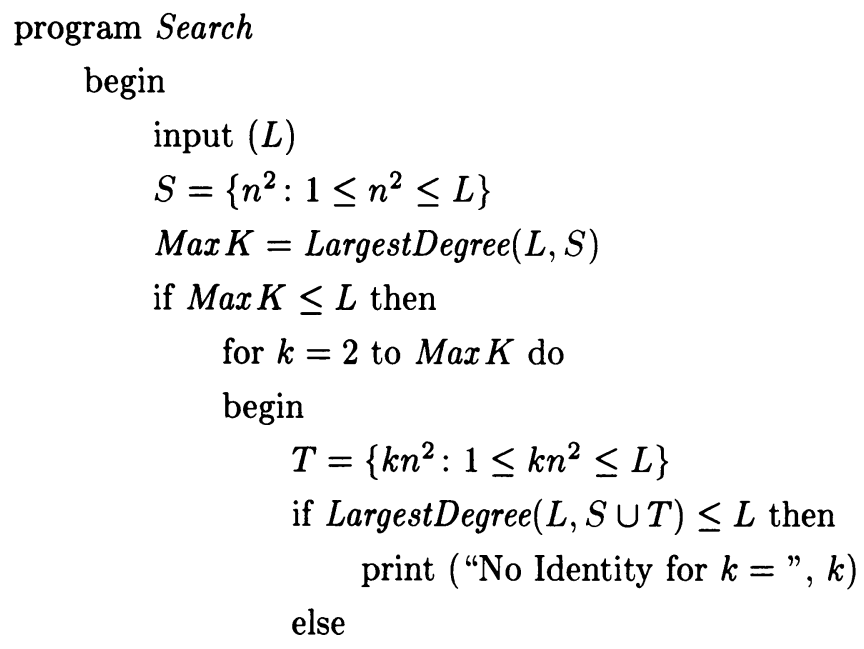

output parameters for those series which factor to degree $L$

end

end.

On execution, with $L=30$, Search finds that $\operatorname{Max} K=15$. This means that all series of the form $1+\sum_{n=1}^{\infty} \delta_{n} x^{n^{2}}$, for any choice of sign $\delta_{n}= \pm 1$, fail to factor into the form on the left-hand side of (45) by degree 15 . Thus, (45) is impossible for $k \geq 16$. Of the values of $k$ between 2 and 15 which fail to factor to degree 30 (all but $k=2,3,5$, and 9), the "longest survivor" is the series $f(x)$ displayed above.

For $k=2,3$, and 5 , the values of $\gamma_{n}, \delta_{n}$, and $\varepsilon_{n}$ determined by the search program agree up to degree 144 with the coefficients of the known identities or the trivial variation of them obtained by replacing $x$ by $-x$. This suggests that the known identities are the only solutions in this case. To prove this, however, would require a much more elaborate investigation of the kind used in [1, Chapter 4]. Note that Theorems 1(a), 3(a) in [3], and (32) above, along with their companions with $x \rightarrow-x$, are the only solutions to (44).

For $k=9$, the values determined by the program for $\gamma_{n}, \delta_{n}$, and $\varepsilon_{n}, 1 \leq n \leq 144$, indicated four basic identities, whose proofs rely on the four functions $Q(6,1)$, $Q_{1}(6,1), Q_{2}(6,1)$, and $Q_{3}(6,1)$. The first two give the identities

$$
\begin{aligned}
Q(6,1) & =(0, \pm 1, \pm 4, \pm 5,6)_{12} \\
& =\sum_{-\infty}^{\infty} x^{3\left(3 n^{2}+n\right)}\left(x^{-3 n}-x^{3 n+1}\right)=\sum_{-\infty}^{\infty}\left(x^{(3 n)^{2}}-x^{(3 n+1)^{2}}\right) \\
& =\sum_{n=-\infty}^{-1}\left(x^{(3 n)^{2}}-x^{(3 n+1)^{2}}\right)+1-x+\sum_{n=1}^{\infty}\left(x^{(3 n)^{2}}-x^{(3 n+1)^{2}}\right) \\
& =1+\sum_{n=1}^{\infty}\left(\delta_{n} x^{n^{2}}+x^{9 n^{2}}\right), \quad \text { where } \delta_{n}= \begin{cases}1, & \text { if } 3 \mid n, \\
-1, & \text { if } 3 \nmid n\end{cases}
\end{aligned}
$$


and the more elaborate

$$
\begin{aligned}
Q_{1}(6,1) & =(0, \pm 1)_{12}[ \pm 4, \pm 5,6]_{12} \\
& =\sum_{-\infty}^{\infty}(-1)^{\left(3 n^{2}+n\right) / 2}\left(x^{(3 n)^{2}}-x^{(3 n+1)^{2}}\right) \\
& =1+\sum_{n=1}^{\infty}\left(\delta_{n} x^{n^{2}}+x^{9 n^{2}}\right), \\
& \text { where } \delta_{n}= \begin{cases}(-1)^{\frac{3 n^{2}-n}{2}-1} & \text { if } n \equiv 0(\bmod 3), \\
(-1)^{\left(3 n^{2}-n\right) / 2} & \text { if } n \equiv 1(\bmod 3), \\
(-1)^{\frac{3 n^{2}-n}{2}+1} & \text { if } n \equiv 2(\bmod 3) .\end{cases}
\end{aligned}
$$

Note in the latter equation that the terms $x^{9 n^{2}}$ cancel when $n$ is odd and double when $n$ is even. Since we can switch the coefficients of $x^{(3 n)^{2}}$ and $x^{9 n^{2}}$ when these terms cancel, there are infinitely many trivial variations of this identity which satisfy (45). The other two basic identities for $k=9$ are obtained from the two above by replacing $x$ by $-x$, or equivalently, by using $Q_{2}(6,1)$ and $Q_{3}(6,1)$.

In a similar way we have searched for identities of the form

$$
\prod_{n=1}^{\infty}\left(1+\gamma_{n} x^{n}\right)=1+\sum_{n=1}^{\infty}\left(\delta_{n} x^{n^{2}-1}+\varepsilon_{n} x^{k n^{2}-1}\right)
$$

where $\gamma_{n}=0, \pm 1$ and $\delta_{n}, \varepsilon_{n}= \pm 1$. (This generalizes Theorems 1 (b) and $3(\mathrm{~b})$ in [3] and (33) above.) The program in this case is the same as Search, but with $n^{2}-1$ and $k n^{2}-1$ being used in the definitions of $S$ and $T$. This time we find that $\operatorname{Max} K=25$ and that all series dead-end by degree 44 except when $k=2,3,4,5$, and 9. As before, for $k=2,3$, and 5 , the values of $\gamma_{n}, \delta_{n}$, and $\varepsilon_{n}$ suggest the known identities are the only ones. For $k=4$, there are four basic identities, stemming from the four functions $T_{0}(16,8), T_{1}(16,18), T_{2}(16,8)$, and $T_{3}(16,8)$. The first is

$$
\begin{aligned}
T_{0}(16,8) & =(0)_{32}[ \pm 8]_{32}=\sum_{-\infty}^{\infty} x^{(4 n+1)^{2}-1} \\
& =\sum_{n=1}^{\infty} x^{(2 n-1)^{2}-1}=\sum_{n=1}^{\infty}\left(x^{n^{2}-1}-x^{4 n^{2}-1}\right) .
\end{aligned}
$$

For the other 3 identities, we use (9), (10), and (11) to obtain

$$
\begin{gathered}
T_{1}(16,8)=(0, \pm 8)_{32}=1+\sum_{n=1}^{\infty}(-1)^{n(n+1) / 2} x^{(2 n-1)^{2}-1} \\
T_{2}(16,8)=(0, \pm 24)_{64}[ \pm 8,32]_{64}=1+\sum_{n=1}^{\infty}(-1)^{(n-1) n(n+1)(n+2) / 8} x^{(2 n-1)^{2}-1}
\end{gathered}
$$

and

$$
T_{3}(16,8)=(0, \pm 8)_{64}[ \pm 24,32]_{64}=1+\sum_{n=1}^{\infty}(-1)^{(n-2) n(n+1)(n+3) / 8} x^{(2 n-1)^{2}-1}
$$

To put these into the form of (46), we need only make sure that the terms $x^{(2 n)^{2}-1}$ and $x^{4 n^{2}-1}$ cancel. 
For $k=9$, there are four basic identities, derived from the four functions $T_{0}(9,6)$, $T_{1}(9,6), T_{2}(9,6)$ and $T_{3}(9,6)$. We give only the first here:

$$
\begin{aligned}
T_{0}(9,6) & =(0)_{182}[ \pm 3]_{18}=\sum_{-\infty}^{\infty} x^{(3 n+1)^{2}-1} \\
& =1+\sum_{n=1}^{\infty}\left(x^{(3 n+1)^{2}-1}+x^{(3 n-1)^{2}-1}\right)=\sum_{n=1}^{\infty}\left(x^{n^{2}-1}-x^{9 n^{2}-1}\right) .
\end{aligned}
$$

The other three identities are obtained in a straightforward manner, similar to that for $k=4$.

Department of Mathematical Sciences

Northern Illinois University

DeKalb, Illinois 60115

Department of Mathematics

University of Arizona

Tucson, Arizona 85721

Department of Applied Mathematics and Statistics

SUNY at Stony Brook

Stony Brook, New York 11794

1. R. Blecksmith, The Determination of Ramanujan Pairs, Ph.D. Dissertation, University of Arizona, 1983.

2. R. Blecksmith, J. BRILlhaRT \& I. Gerst, "A computer-assisted investigation of Ramanujan pairs," Math. Comp., v. 46, 1986, pp. 731-749.

3. R. BLECKSMITH, J. BRILLHART \& I GERST, "Parity results for certain partition functions and identities similar to theta function identities," Math. Comp., v. 48, 1987, pp. 29-38.

4. R. Blecksmith, J. BRILlhart \& I. Gerst, "Some infinite product identities," Abstracts Amer. Math. Soc., v. 8, 1987, p. 439.

5. L. Carlitz \& M. V. Subbarao, "A simple proof of the quintuple product identity," Proc. Amer. Math. Soc., v. 32, 1972, pp. 42-44.

6. J. A. Ewell, "Completion of a Gaussian derivation," Proc. Amer. Math. Soc., v. 84, 1982, pp. 311-314.

7. J. A. EWELL, "Some combinatorial identities and arithmetic applications," Rocky Mountain J. Math., v. 15, 1985, pp. 365-370.

8. G. H. HARDY \& E. M. WRIGHT, An Introduction to the Theory of Numbers, 4th ed., Oxford Univ. Press, Oxford, 1965. 